\title{
Towards multi-domain knowledge transfer in engineering analyses and simulations based on virtual prototypes
}

\author{
Zoltán Rusák • Imre Horváth • Ferruccio Mandorli
}

Published online: 9 June 2013

(C) Springer-Verlag London 2013

The use of virtual prototypes in product development has been proliferating rapidly in the last two decades. New computational methods and tools have been developed to generate virtual prototypes for various engineering analysis, simulation and optimization tasks. Virtual prototypes can represent several aspects of products, including among others; geometric and structural properties, physical operations and behavior, and production, use and maintenance processes. The development of methods and tools is continuously expanding towards new application domains and users are getting more and more involved in testing virtual prototypes of products. Numerical computations are getting more accurate and reliable. Through the analyses and simulations of virtual prototypes, design engineers are now able to ensure that a product will possess exactly the desired functions, properties, behaviour, quality and cost.

In the hope of new opportunities, multi-disciplinary, transdisciplinary and trans-domain knowledge transformation has become one of the central topics of scientific research in virtual prototyping. Typical examples of multi- and transdomain knowledge transformation are based on: (i) generalization of methods (e.g., design of computer experiments), (ii) introduction of novel technologies to enable simulation of complex physical phenomena (e.g., multiple contact in ropes), (iii) development of multi-physics solvers that facilitate the analysis of multi-faceted systems, and (iv) integration of interactive virtual testing of concepts into the product

\footnotetext{
Z. Rusák $(\bowtie) \cdot$ I. Horváth

Delft University of Technology, Delft, The Netherlands

e-mail: Z.Rusak@tudelft.nl

F. Mandorli

Università Politecnica delle Marche, Ancona, Italy
}

validation processes. In the field of computer-aided engineering analysis and simulation, multi-disciplinary transformation of knowledge involves the following activities: (1) identification of opportunities for integration of knowledge of multiple disciplines, (2) exporting knowledge among various mono-disciplinary applications, and (3) combining monodisciplinary knowledge to enable multi-disciplinary simulation and optimization. It has to be noted that the mono-disciplinary analysis and simulation methods are also further refined. The new methods and tools allow us to model, simulate and optimize the operations of complex engineering systems, such as internal combustion engines, industrial process plants, multi-product packaging systems, smart robotic systems, etc.

In this Special Issue, we provide examples of multi-, and trans-disciplinary and trans-domain knowledge and numerical computation technology transfer in the field of engineering analysis and simulation, in addition to presenting results of the efforts towards enhancing mono-disciplinary analysis and simulation approaches. Furthermore, we intend to show that the methods and tools proposed by the contributing authors are not only significant from an academic point of view, but are also valuable for the industrial practice. The selected papers cover a wide range of application fields, indicating the importance of multi-domain knowledge enrichment and transfer. Originally, the papers were presented at the Ninth International Symposium on Tools and Methods of Competitive Engineering (TMCE 2010), that was held in Ancona, Italy, from 12 until 16 April, 2010. The TMCE 2010 Symposium was jointly organized by the Università Politecnica delle Marche, Italy, and the Delft University of Technology, the Netherlands.

The papers have been thematically arranged in this special issue. The first five papers are representative examples of knowledge enrichment. They focus on mono- 
disciplinary analysis, and simulation problems of complex products and manufacturing processes. The next five papers present various approaches to combining knowledge from multiple disciplines and domains. This is needed, for instance, when human factors need to be considered in virtual usability testing of engineering control mechanisms, or when the objective is to create one single software platform for concurrent investigation of mechanical, hydraulic and electronic subsystems.

Analysis and simulation of worm gear design and manufacturing processes is an important field of activity for the CAE research community already for decades. The first paper, written by Dudás under the title "Modeling and simulation of a new worm gear drive having point-like contact", argues that novel worm gear drives and innovative gearing solutions require, on the one hand, a more rigorous theoretical underpinning and, on the other hand, a simplified design workflow. The Surface Constructor software, developed by the author, facilitates the generation of gear surfaces as well as the visualization of contact areas. It is based on the Reaching Model surface generation theory that facilitates the generation of conjugate surfaces and surfaces with point like contacts. Using three levels of representation (i.e. symbolic, numeric and visual), the software is able to guide the user through a very simple workflow and enables them to design worm gear mechanisms and to generate their virtual prototypes with high accuracy. Though the software has not been tested in a real life design processes, it seems to be capable of supporting a concurrent engineering workflow, in which product designers and production engineers can concurrently design worm gear mechanisms. A possible improvement of the current solution can be the incorporation of an optimization method to reduce the time needed to for the analysis and simulation of alternative concepts of worm gear drives.

The second paper, entitled "Design curve determination for two-layered wire rope strand using p-version finite element code", is written by Beleznai and Páczelt. This paper presents a new method for designing and testing rope structures under axial loads. The authors have developed a non-linear contact model based on Hertz' theory, in order to be able to analyze interwire motions, and the contact and friction effects, by taking into account homogenous, isotropic, and linear-elastic material properties. For the investigation of these multi-physical phenomena, a specific p-version finite element and a numerical solver have been implemented. The software helps designers to develop and test virtual prototypes of rope structures. The virtual prototypes can be defined by a few geometric, load and constraint parameters. The software also offers a sensitivity analysis tool which helps explore the design space of rope structures, and determine the optimal geometric parameters. The proposed solution offers not only a more efficient computation method, but also is easy to use. It can be integrated into commercial finite element software. The proposed solution could possibly be extended with a methodological framework that would systematically guide users in the exploration of the design space of rope structures.

The third paper, "Multi-objective topology optimization through GA-based hybridization of partial solutions", submitted by Cardillo et al., proposes to combine genetic algorithms (GAs) and optimality criteria (OCs) algorithms to achieve extra benefits in topology optimization of mechanical structures. As shown by the authors, OCs are more computational efficient and effective than GAs. By contrast, GAs have higher efficacy in finding the global optimum. The proposed hybrid approach: (1) decomposes multi-objective optimization problem into mono-objective problems, (2) uses OCs to determine the density distribution of materials in the mechanical structure that is subjected to a given mechanical load (i.e. applies a normalization to the range $0-1$ interval for the stress values caused by the applied load), (3) filters out under-loaded elements by applying given threshold in the process of refining the topological solution space, (4) applies structural analysis on the refined topological structure and determines new values for density threshold, and (5) repeats step 3 and 4 until the global optimum is reached by a genetic algorithm. The effectiveness of the hybrid approach is demonstrated via several case studies. It has been found that the hybrid approach is not only effective, but also offers more flexibility in exploring design space and in finding possible solutions which fall outside the Pareto frontier. The hybridization approach discussed in the paper opens up new research opportunities in understanding context-depended application of optimization methods and the possibilities of combining them in various engineering applications.

In the paper "Integrated metal forming and vibration analysis of sheet metal parts" Echempati and Fox systematically analyze two issues of forming sheet metal parts, namely material thinning and vibration. The objective of their work was to optimize the design and production parameters that influence the forming process of sheet metal products from these aspects. They used the Hyperform and Dynaform/LSDYNA software tools to develop various regression models in the methodological framework of design of experiments (DOE) and response surface method (RMS). Benefiting from DOE, the significance of factors influencing springback, such as sheet metal thickness, friction coefficient, and press tonnage, of the regression models, has been determined. By applying RMS with two independent variables, regression equations were developed to determine the effects of sheet metal thickness and friction coefficient on the springback. The authors argue that they found significant difference in terms of the 
reliability and accuracy between the regression models computed from the results obtained by Dynaform/LSDYNA and Hyperform. The paper also presents a case study for vibrational analysis of the instrument panel of a car. Overall, the paper gives a good example of how CAE tools can support design of sheet metal products and their production processes through virtual prototypes and fast and accurate analysis and optimization methods. What can be expected in the near future is that tools will be able to incorporate many more additional design and production parameters (e.g., punching speed, binder force) in the optimization of sheet metal products and forming processes.

In their paper, entitled "Computer aided engineering for sheet metal forming: Definition of a springback quality function", Campana et al. propose an approximation method for estimation of the springback of high-strength sheet metal materials. The authors consider the remaining spring force as an additional physical effect and combine it with the vibrational forces distributed over the nodes. The springback quality function is determined as equilibrium between the nodal force field applied on the finite element model of the deformed sheet metal component and the linear combination of the nodal force fields related to $\mathrm{N}$ natural modes of vibrations of the component. Having tested this approach in several complex applications, the authors found that the proposed approximation method shows coherent and consistent results with the experimental data. Although the proposed approximation method is simple and elegant for estimating the springback of sheet metal components, its accuracy highly depends on the errors of the nodal force field applied in the finite element analysis model.

In the sixth paper, entitled "Design of computer experiments applied to modeling of compliant mechanisms for real-time control", Acosta et al. propose to adapt the design of computer experiments (DOCE) methodology to modeling and evaluation of compliant mechanisms under quasi-static conditions. As argued, DOCE can be used for pre-defining and testing the range of design parameters of compliant mechanisms, which can be further refined by using the design of experiment method (i.e. by completing actual physical tests). The proposed DOCE methodology facilitates the modeling of compliant mechanisms that have lumped or distributed components with simple or complex geometry. The strengths of the proposed solution are that: (i) it is general enough to cover both lumped and distributed compliant mechanisms, (ii) the obtained input-output model is typically simple enough to be used in real-time control, and (iii) experimentation with physical prototypes is replaced by computer simulations; thereby the costs of product development are reduced. The first test results have shown that trans-domain application of the DOCE methodology is beneficial since it provides sufficient accuracy for designing compliant mechanisms considering their operating points. However, a more extensive validation and verification of the proposed methodology seems to be necessary. It can be extended with multi-physics principles in order to handle non-ideal situations, where the deformation caused by wear, temperature change and hysteresis could be considered concurrently.

The seventh paper entitled "A comparison of simplex and simulated annealing for optimal designing of a new rear underrun protective device", written by Ingrassia et al., compares the applicability of two optimization methods for designing and optimizing complex vehicle structures. It presents the basic principles, as well as the advantages and disadvantages of simplex-based and simulated annealingbased optimization methods. The capability and limitations of these methods have been compared within a case study, which focused on the optimization of a new underrun protection system for trucks to prevent underrun of passenger cars during accidents. They also made effort to optimize the dynamic performance of the underrun protection system, and to manage a large number of state and design variables. Even though both methods are very effective in finding the optimum solution for this dynamic structure, the authors argue to use simulated annealing for exploration of and narrowing down the design space, and to apply simplex method for finding the global optimum. The paper suggests that an appropriate combination of optimization methods can have a great potential in applications domains other than automotive engineering.

In the paper entitled "A virtual reality approach for usability assessment- case study on a wheelchair-mounted robot manipulator", Di Gironimo et al. propose to combine multi-criteria decision analysis with Saaty's analytic hierarchy in order to enable the assessment of product usability by means of virtual prototypes. The proposed approach takes into account objective and subjective aspects of userproduct interaction. It is also capable to distinguish negative usability experiences that are caused by the limitations of the applied virtual reality environment from the actual negative usability experiences revealed by the virtual prototype of the designed product. The paper suggests that successful integration of multidisciplinary knowledge, that is, combination of knowledge of human sciences (e.g., ergonomics) and engineering sciences (e.g., computer science, mechatronics and control engineering) creates a fertile ground for future research into methodological and computational aspects of virtual prototyping. Furthermore, it also provides an opportunity to extend the proposed design methodology to domains of application other than assistive technologies for disabled.

The paper "Reliable behavior simulation of product interface in Mixed Reality" by Bruno et al. introduces a 
new approach for interactive usability testing of virtual prototypes of product concepts. The authors argue that designing of products and developing their mixed reality prototypes in a team of product designers, control engineers and virtual reality operators, using an integrated software platform, results in more efficient testing processes. It is shown in the paper that this integrated software platform allows designers and control engineers to quickly modify product concepts and adapt interfaces to the needs of individual users during usability testing. Furthermore, it is argued that the proposed mixed reality model is not only beneficial in combining multi-physical effects in the virtual world, but can also offer a platform for using the physical effects of the real world, for instance, for providing tactile feedback to the user. Overall, the proposed approach facilities the exploration of alternative design concepts, and, compared to pure virtual reality approaches, it is more efficient and reliable in usability testing.

The last paper, "Virtual engineering at work: The challenges for designing mechatronic products" by $\mathrm{Au}-$ weraer et al., gives an outlook into the near future. Besides offering a state of the art review on the methods and tools of mechanical system design and control design, it also deals with emerging product simulation software. The paper exemplifies the importance of multi-disciplinary knowledge and technology transformation by addressing two challenges that are associated with the next steps of the evolution of virtual prototyping. First, it recognizes the need for multi-physics simulation in the context of designing and testing intelligent products having thermal, hydraulic, mechanical, and electrical components. Secondly, it identifies the need for integrating system design and control design processes through heterogeneous simulation methods, which enables a so-called all-inclusive evaluation of system behavior, which covers interaction of the components, the subsystems and the environment. The authors propose to apply a scalable approach that not only enables multi-physics simulation, but also allows us to combine 3D geometric simulations (multi-body, finite element) and 1D functional and control simulation (representing the logic of product operation). The paper also discusses the AUTOSAR standard as demonstrative examples of the above concepts and presents several cases from the automotive industry.

We expect that through this composition of papers, we have managed to provide good examples of new computational methods and tools that are both significant academic results and beneficial means for computationintensive industrial product design. We also expect that we have managed to demonstrate the best research practices for fellow researchers by means of these papers. We are grateful to all authors for their contribution to this special issue. We do appreciate their efforts and nice collaboration. We are also indebted to our reviewers who helped us to increase the scientific/professional value and quality of the papers. 